\title{
LOCAL STATISTICS IN NATURAL SCENES PREDICT THE SALIENCY OF SYNTHETIC TEXTURES
}

G Tkačik ${ }^{*}$, JS Prentice*, JD Victor ${ }^{+}$,V Balasubramanian*

"Dept. of Physics and Astronomy, University of Pennsylvania

Institute of Science and Technology Austria

+Dept. of Neurology and Neuroscience, Weill Cornell Medical College

\section{Introduction}

Psychophysical measurements show that human observers can distinguish:

(a) textures in which pixels are independently drawn from different histograms if the histograms differ in mean, or variance, or "blackshot" statistic [1]

(b) textures with black-and-white pixels, in which there is only 4th order correlation between certain, but not all, geometrical arrangements of pixels ("gliders") [2]

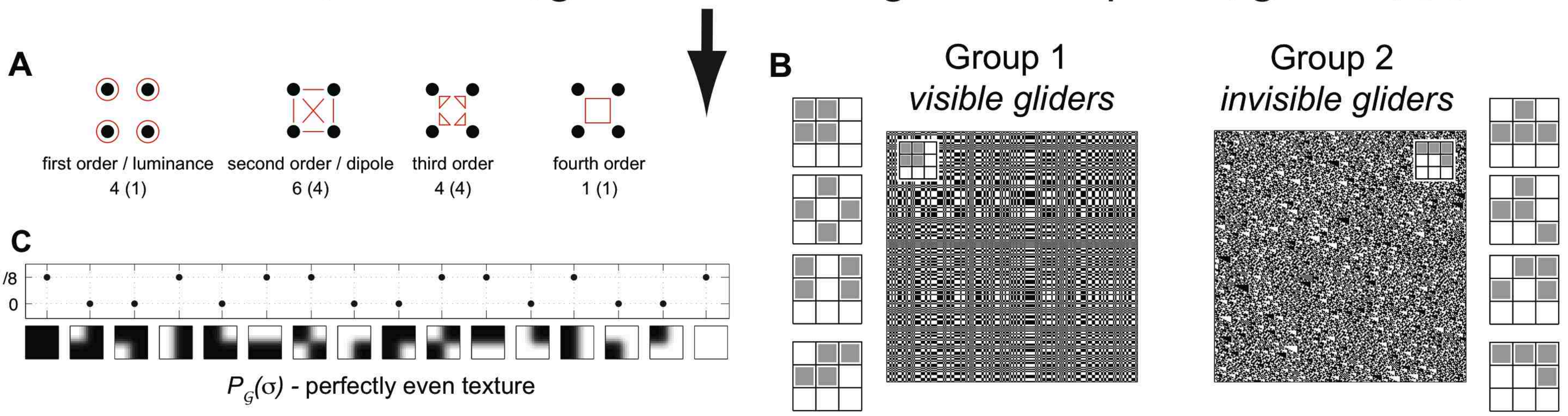

Difference in

histogram..

mean

variance

blackshot

(not easily observable statistic)

Here we ask if these results can be explained by the statistics of natural scenes. Does texture visibility correlate with their occurrence in natural scenes? Does the efficient coding hypothesis extend to higher-order statistics and to the central brain?

\section{IID texture in natural scenes}

1. Discretize the image into 16 grayscale histogram-equalized levels, sample conditional pixel histograms
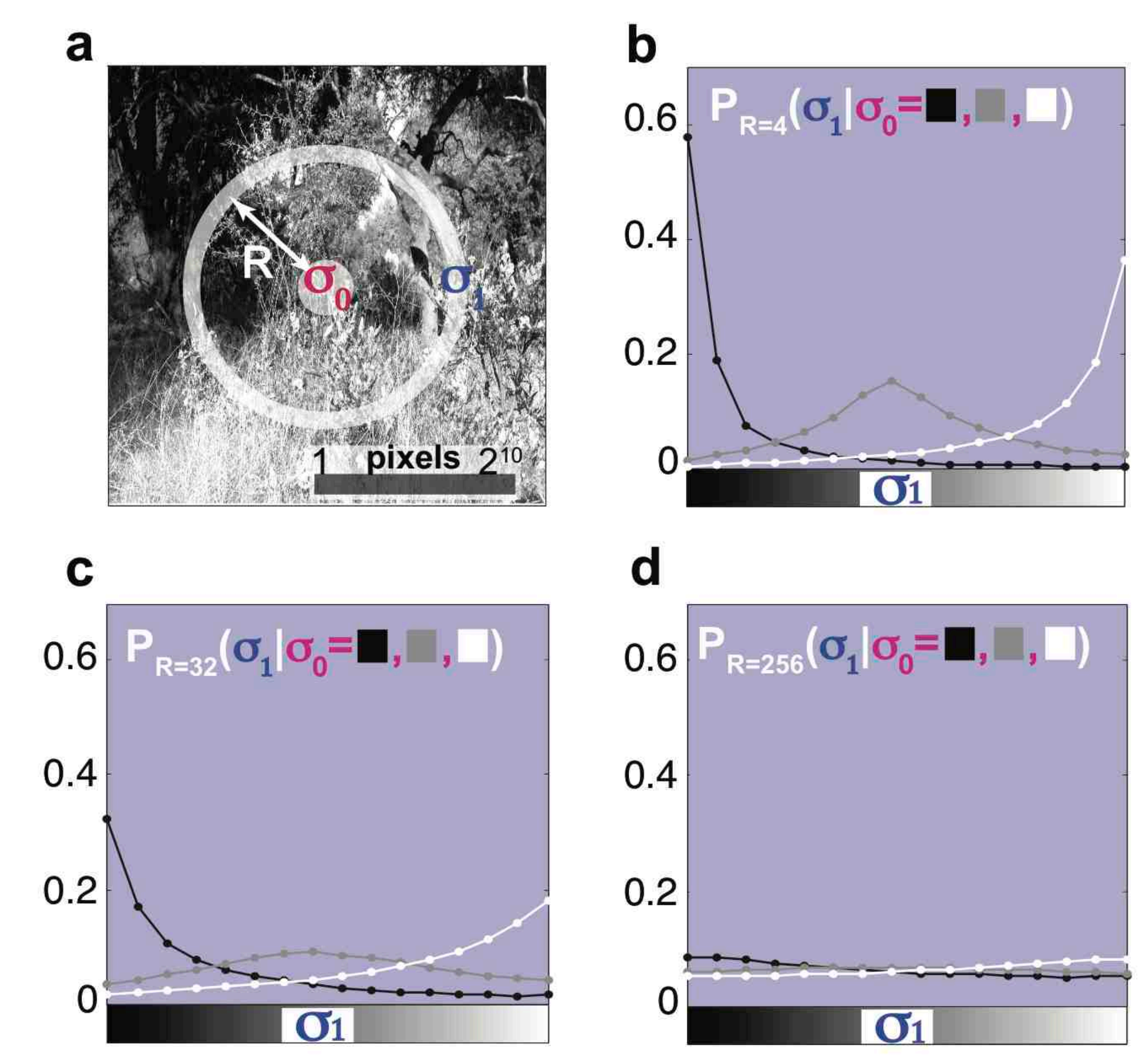

Note the asymmetry between black and white central pixels
2.PCA on the histograms finds 3 dominant components that span the same subspace as mean, variance and blackshot
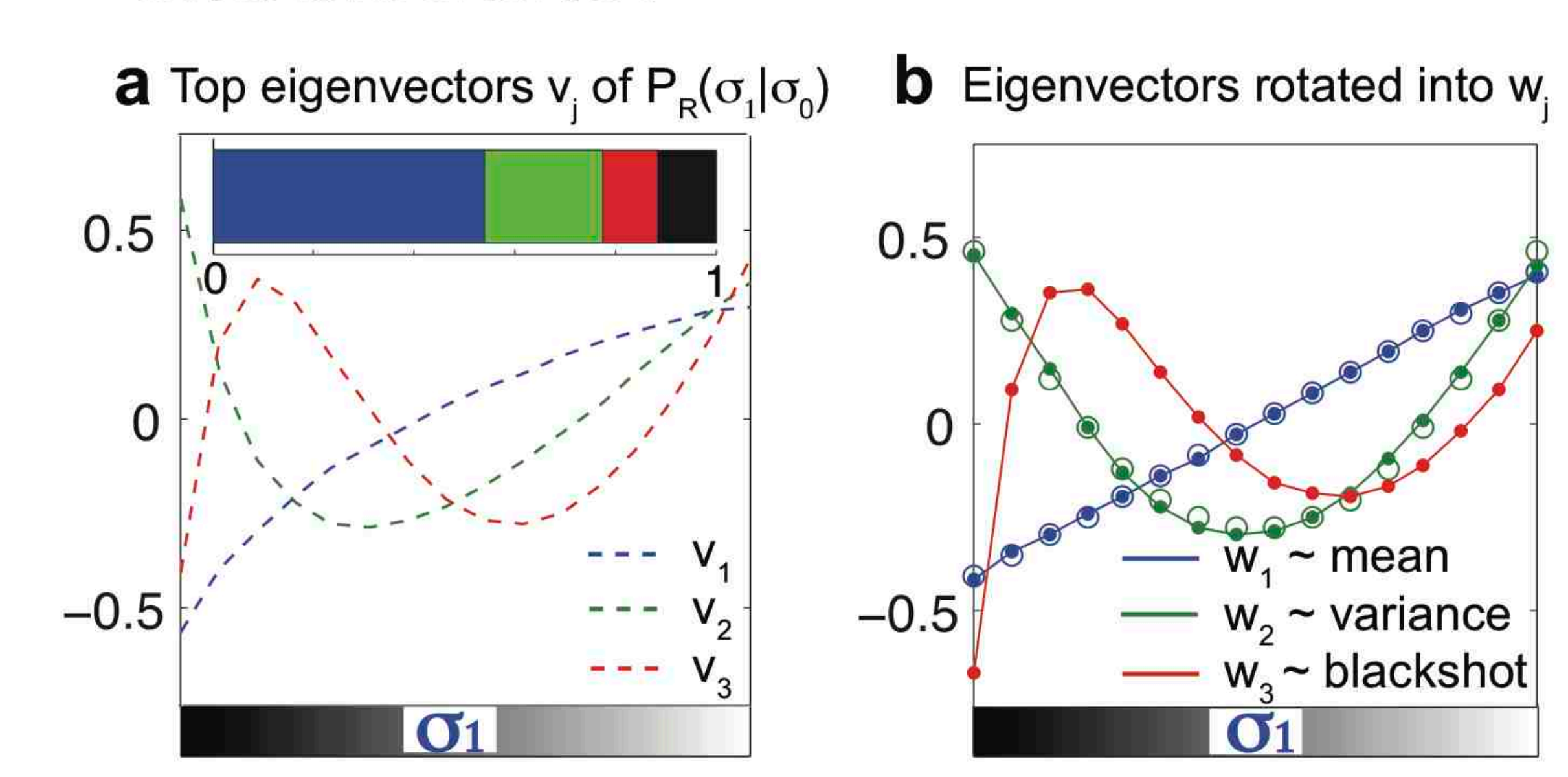

Human observers perceive difference in synthetic IID texture if the histogram of pixel intensities differs from uniform along one of the 3 axes: mean, variance, blackshot.

Here we find that in natural scenes most of the variance in pixel intensity histograms lies along the same 3 axes. These results are robust with respect to various sampling strategies.

\section{Conclusions}

Human visual system can detect (only) those correlations that are present in natural scenes. We find that this claim applies not only to 2 nd order image statistics and the linear filtering operations in sensory periphery, but also to higher-order correlations in the scenes and the nonlinear mechanisms in the central brain.

\section{References}

Tkacik G, Prentice JS, Victor JD, Balasubramanian V (2010) Local statistics in natural scenes predict the saliency of synthetic textures. PNAS 107: 18149-54

C Chubb, MS Landy, J Econopouly (2004) Vision Res 44:3223-33

JD Victor, C Chubb, MM Conte (2005) Vision Res 45: 311-328

\section{$4^{\text {th }}$ order textures in natural scenes}

1. Whiten and binarize the image. Slide each glider shape across image patches of size $\mathrm{R}$, accumulate histogram of $2^{4}$ glider colorings. Compute how much of the histogram structure is explained by pairwise, triplet and quadruplet correlations.
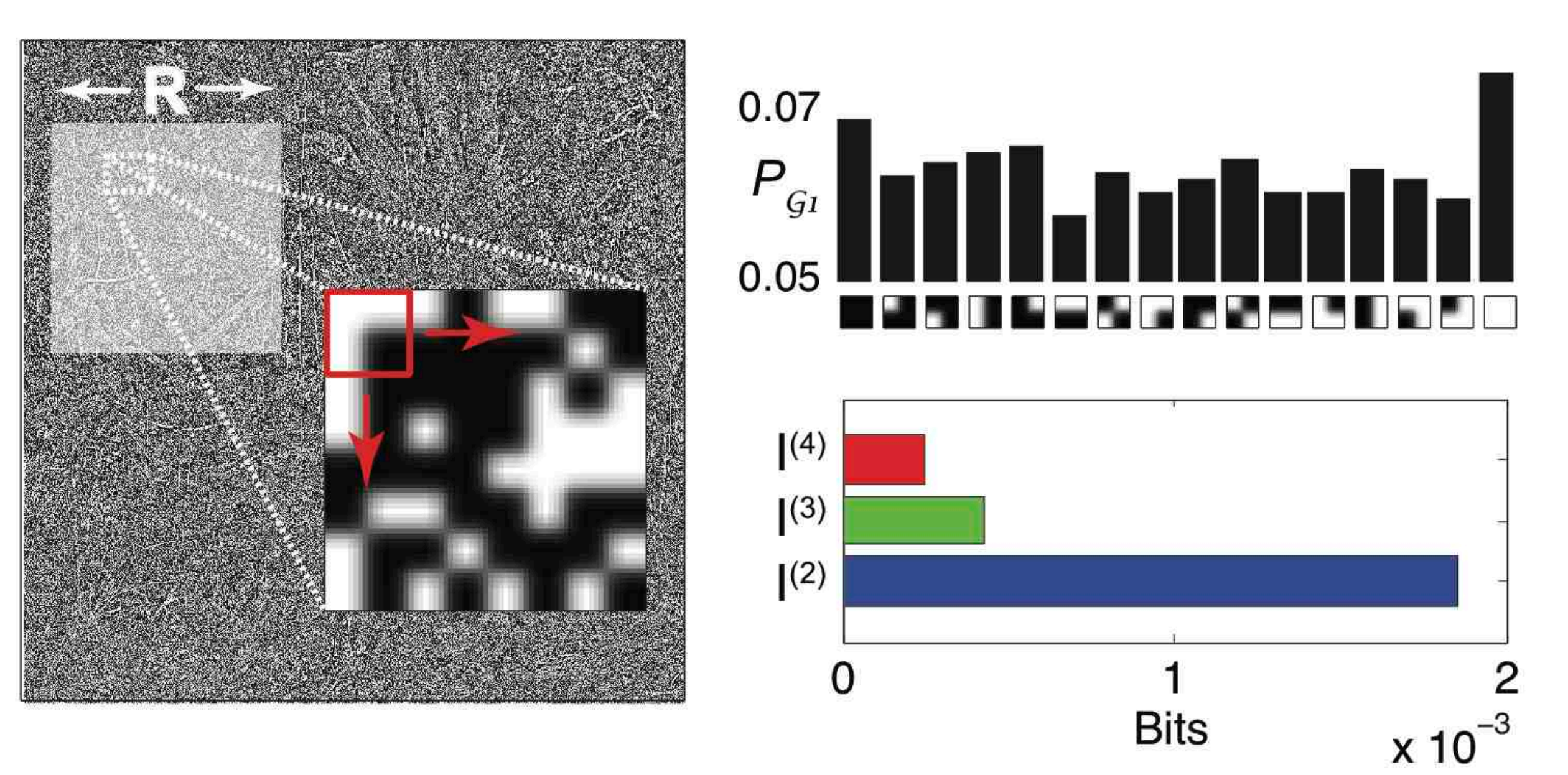

2. As patch size $\mathrm{R}$ grows, $4^{\text {th }}$ order correlation as measured by I(4) and parity $\alpha^{(4)}$ grows in Group I but not Group II gliders. Human observers perceive only those $4^{\text {th }}$ order synthetic textures that have statistical structure present in nature.

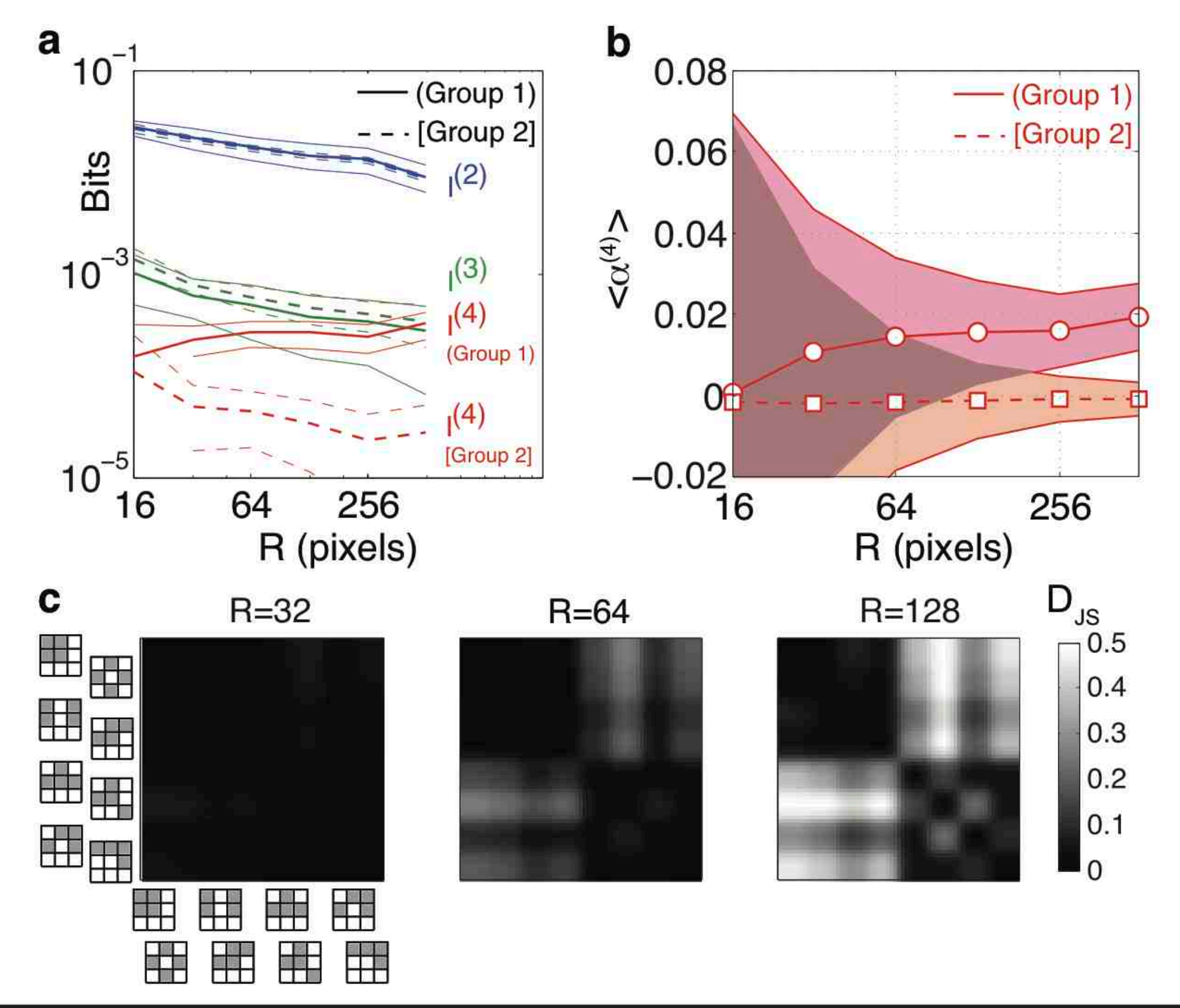

